\title{
Automatic generation of checklists from business process model and notation (BPMN) models for surgical assist systems
}

https://doi.org/10.1515/cdbme-2020-0005

\begin{abstract}
Checklists are a valuable tool to ensure process quality and quality of care. To ensure proper integration in clinical processes, it would be desirable to generate checklists directly from formal process descriptions. Those checklists could also be used for user interaction in context-aware surgical assist systems. We built a tool to automatically convert Business Process Model and Notation (BPMN) process models to checklists displayed as HTML websites. Gateways representing decisions are mapped to checklist items that trigger dynamic content loading based on the placed checkmark. The usability of the resulting system was positively evaluated regarding comprehensibility and end-user friendliness.
\end{abstract}

Keywords: business process model and notation (BPMN); checklists; surgical workflow; user interface.

\section{Motivation}

To avoid adverse events during surgery, the use of checklists is suggested as a helpful tool. Despite the evidence that checklists can significantly improve the quality of care, they have not yet effectively changed clinical practice. One reason for this is the additional workload due to poor integration into daily practice and routine.

The OR-Pad research project at Reutlingen University is developing a prototype that provides context-relevant information near the surgeon. To recognize the current context of a surgery, a situation recognition based on process models is used. The underlying process model offers the possibility to support the surgeon in the execution

\footnotetext{
*Corresponding author: Claudia Ryniak, Informatik, Reutlingen University, FG CaMed, Alteburgstr. 150, Reutlingen, Germany, E-mail: Claudia.Ryniak@Reutlingen-University.DE

Oliver Burgert, Informatik, Reutlingen University, FG CaMed, Alteburgstr. 150, Reutlingen, Germany,

E-mail: Oliver.Burgert@Reutlingen-University.DE
}

of the process steps by displaying relevant information for each surgical phase.

The visualization of the process steps by dynamic checklists was planned. Furthermore, checklists can be used as input for the situation recognition systems since they can be used as input provided by a human expert.

In this work, a system is developed that can automatically convert Business Process Model and Notation (BPMN) process models into dynamic checklists.

\section{State of the art}

\section{Checklists}

Lessing et al. [1] define checklists as working tools that serve as reminders or structure processes and actions in a consistent way. In stress and exceptional situations, they can be helpful to prevent memory errors and support decision-making. A checklist contains all the relevant aspects of a particular work process. They are uniformly arranged and allow the user to check the presence or absence of each of the listed elements [2,3]. Although a checklist appears restrictive by its structure, it allows the focus to be on the actual work and relieves the brain. Through the consistent use of checklists, processes can be improved and errors are avoided. The simultaneous documentation in the checklist makes it possible to track the performed work steps [3].

In the medical context, a checklist is called a memory aid, which consists of a detailed list of tasks and during a specific process to be done. A checklist divides complex tasks into simpler components and lists the essential or critical steps of a process. It also determines which tasks can be delegated to other persons and encourages the consistency of the service. [4] The probably best-known checklist in the surgical domain is the World Health Organization (WHO) checklist [5]. The Surgical Patient Safety System (SURPASS) was developed by the Academic Medical Center (AMC) to reduce the mortality rate and complications during surgery. This checklist standardizes all procedures of a surgical process and defines responsibilities and specific controls. In contrast to the WHO checklist, the control of the 
process begins with the admission of the patient and not just shortly before the surgery [6, 7]. Manataki et al. [8] developed a systematic, computer-aided approach for the creation of structured checklists for intra-clinical transfer. A three-phase methodology is proposed, in which hospitalinternal transfer processes are first designed and combined into workflows. These are then checked by machine and finally translated into a series of checklists that are intended to support hospital staff while maintaining the dependencies between different tasks.

Workflow supporting systems are the research field of several workgroups, but most of them focus on automatic situation recognition to determine the current state of the surgery [9-11]. In [12] a "surgical procedure manager" is used to guide the surgeon through an endonasal procedure using pre-defined checklist items, which are confirmed by a footswitch.

We did not find any system describing the automatic generation of medical checklists based on structured process descriptions like BPMN.

\section{Process modelling}

Business process models are used to describe real-world processes in graphical form. They use specific elements for process representation and contain rules for defining the elements among themselves. There are many different languages for modeling business processes and workflows [13]. BPMN is a standard for modeling and automating business processes. Other standards that are related to BPMN and complement it: Case Management Model and Notation (CMMN) for managing unstructured activities and Decision Management Notation (DMN) for managing decisions [14]. A guideline on how to model surgical procedures based on those standards can be found in [15].

\section{Methods}

To derive checklists from BPMN, we developed a schema for the transformation of BPMN elements to checklist items (see Table 1). Elements that are similar in their purpose and meaning were compared and contrasted.

BPMN is serialized as an XML file, so we read the XML-files describing the Process with the help of an XML parser. A Document Object Model (DOM) is created which represents the BPMN process elements in a tree data structure. It contains all information of the XML file and makes them available to the system. All relevant information is read from the DOM document and sorted in the specified order. Finally, HTML elements are generated by which the final checklist is combined.

Sequential activities are sorted in order of the process flow. Start event and end state are included. Subprocesses are represented as tabs
Table 1: Transformation of business process model and notation (BPMN) elements to checklist items.

\begin{tabular}{|c|c|}
\hline $\begin{array}{l}\text { BPMN } \\
\text { element }\end{array}$ & Checklist item \\
\hline Subprocess & Checklist \\
\hline Activity & Checklist task \\
\hline Event & Status, checklist, symbol, text element \\
\hline Gateway & $\begin{array}{l}\text { AND: limited influence on checklist, order } \\
\text { XOR: indentation, dynamic loading depending on } \\
\text { selection/decision }\end{array}$ \\
\hline $\begin{array}{l}\text { Sequence } \\
\text { flow }\end{array}$ & Order, priority \\
\hline Annotation & Symbol, for XOR gateway: display question \\
\hline Pool \& lane & Checklist \\
\hline
\end{tabular}

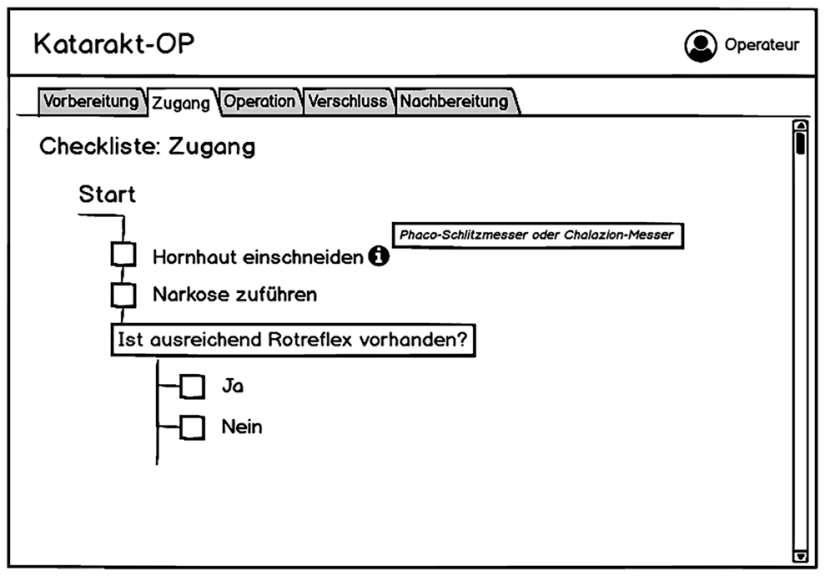

Figure 1: Wireframe design concept for checklist items representing business process model and notation (BPMN) elements.

for easier handling if one person has to work with different checklists. BPMN swimlanes are represented as roles and after selecting the role, the respective tasks are shown in the checklist view. Exclusive gateways (decisions) are shown as a text label presenting the decision to be made and the possible answers below. Depending on the answer, the next checklist items are generated (see Figure 1). Supplementary information can be added to a task and it is shown as tooltip indicated by an (i)-symbol.

If a checklist task is confirmed, it is colored in green to indicate that it has been completed. Checklist tasks that have been skipped are colored in orange. This is to indicate to the user that a checklist task has not been completed. Orange was chosen because it should only provide an indication so that the user can decide for himself whether the checklist task is relevant to his activity or not. The status of the checklist tasks can be changed at any time.

We used the BPMN model of cataract intervention [15] created with the Camunda Modeler ${ }^{1}$ for a first user study. The BPMN model was

$1 \mathrm{https} / /$ camunda.com/de/products/modeler/. 


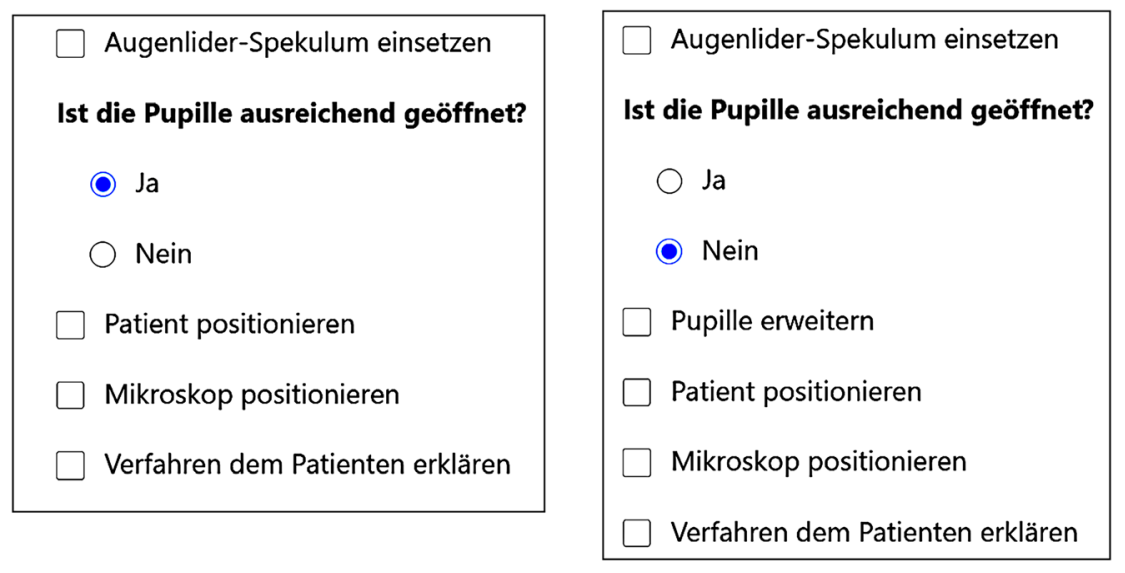

Figure 2: Dynamic loading of checklist items based on answers given in the checklist. built hierarchically and consisted of five phases, which are represented as tabs in the final view. Each phase contained activities and flow control elements that were converted by our software to checklist items according to the method described above.

For usability evaluation, 10 people were reviewing the BPMN models and the corresponding checklist of the intervention. They had to perform several tasks on the BPMN representation and the checklist while using a think-aloud technique to document their mental representation of the process. They answered a questionnaire focused on comprehensibility and user-friendliness. Furthermore, clinical experts were interviewed regarding the clinical applicability of the system.

\section{Results}

\section{System prototype}

The basic structure of the prototype follows the general structure of a web application according to the clientserver model. On the client side, HTML 5 and CSS3 are used to display the page. JavaScript is used to implement the client- and server-side logic. The system was tested on a BARCO medical tablet $\mathrm{PC}^{2}$. The tabs were moved from the top of the screen to the left to leave more vertical room for the checklist items. Figure 2 shows the system running in full-screen mode of the web browser on the tablet PC. Figure 3 shows the dynamic loading of new checklist items according to the decision gateway in the BPMN model.

To test the transformation from BPMN to checklists, 24 different BPMN process models were used. These include 15 BPMN process models of cataract surgery and nine BPMN process models from orthopedics (cervical spine stiffening, claw toe correction, and total hip

2 MUIP-2112. endoprosthesis). All BPMN models have been transformed correctly.

\section{Usability test}

Ten people participated in the usability evaluation; none of them was a clinician. Three people had a background in BPMN, two in the medical domain, two in BPMN and the medical domain, and three had no prior knowledge at all.

The comprehensibility results for BPMN and checklist representation are shown in Table 2. Another crosscorrelation with BPMN experience showed that checklists are easier to understand especially if BPMN models are an unfamiliar representation. One test subject found BPMN to be more comprehensible than checklists, six rated checklists as more comprehensible than BPMN, and three rated both representations equal.

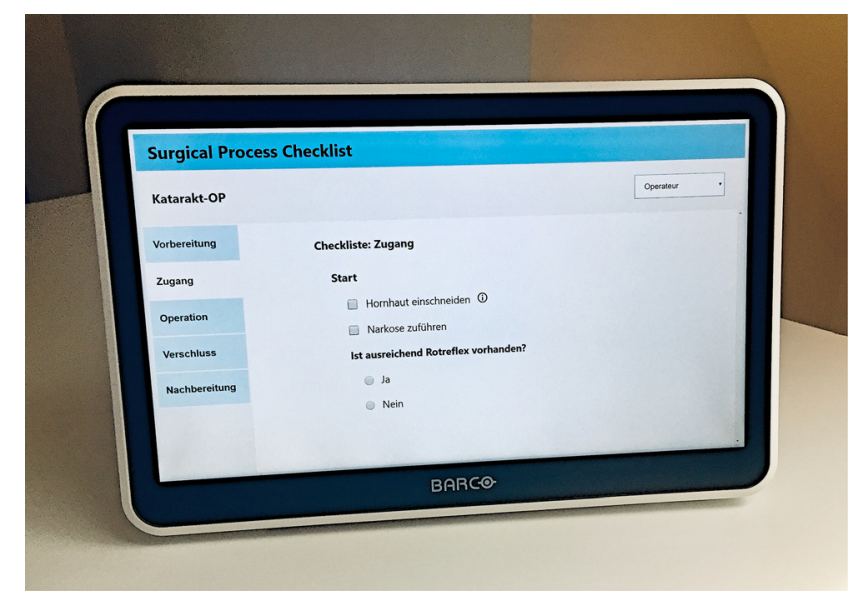

Figure 3: Automatically generated surgical process checklist running on a medical tablet $\mathrm{PC}$. 
Table 2: Comprehensibility of BPMN and checklists.

\begin{tabular}{lrr}
\hline Comprehensibility & \multicolumn{2}{r}{ Number of test persons } \\
\cline { 2 - 3 } & BPMN & Checklist \\
\hline Very easy & 3 & 5 \\
Easy & 4 & 5 \\
Neutral & 2 & 0 \\
Difficult & 1 & 0 \\
Very difficult & 0 & 0 \\
\hline
\end{tabular}

The screen design and user interface were evaluated using a Likert scale ranging from 1 for "is true" to 5 "is not true". The only usability metrics not rated "1" were "Working through the checklist tasks is simple and not timeconsuming" rated with 2 ("partially true") and "Working through the checklist requires little interaction" rated with 1.5 .

The interview with the clinical expert was very positive regarding the overall system, but it was stated that the checklists should focus on relevant parts, clinical context information could be added to checklist items, and that the checklists should integrate seamlessly in the surgery since the surgeons are focused on the intervention. It could be an option to delegate the checking of items to a surgical assistant.

\section{Discussion}

We demonstrated that BPMN models representing surgical interventions could be automatically transformed into surgical checklists resulting in a more intuitive representation, especially for non-BPMN-experts. Therefore, we assume that such a transformation can be of value when clinical processes are discussed in an interdisciplinary team.

The resulting user interface was rated as appropriate, but the interaction effort is significant and therefore we do not recommend using such a system in an intraoperative setting where the checklist must be filled in by a surgeon. If there is supporting staff available, such checklists could be integrated.

Nonetheless, the full potential of checklists lies in the integration of checklists as input and output for situationaware systems, where a situation recognition can check items automatically and the checklist is presented as a front end for such a workflow guided system. Furthermore, it could be used as input especially for decisions that cannot be detected by a situation recognition automatically.
Author contributions: All authors have accepted responsibility for the entire content of this manuscript and approved its submission.

Research funding: This project is supported by the Ministry of Science, Research and the Arts Baden-Württemberg and the European Fund for Regional Development.

Conflict of interest: Authors state no conflict of interest. Informed consent: This article does not contain patient data.

Ethical approval: This article does not contain any studies with human participants or animals performed by the authors.

\section{References}

1. Lessing C, Francois-Kettner H, Jonitz G, Bauer H, Schrappe M. Checklisten im OP - ein sinnvolles instrument zur verbesserung der patientensicherheit? Perioperative Medizin 2010;2:179-86.

2. Hales B, Terblanche M, Fowler R, Sibbald W. Development of medical checklists for improved quality of patient care. Int J Qual Health Care 2008;20:22-30.

3. KVWL. KPQM. KV-praxis-qualitätsmanagement; 2017. Available from: https://www.kvwl.de/arzt/qsqm/management/ handbuch/kpqm_5_30.pdf [Accessed 11 May 2020].

4. Bernstein PS, Combs CA, Shields LE, Clark SL, Eppes CS. The development and implementation of checklists in obstetrics. Am J Obstet Gynecol 2017;217:B2-6.

5. World Health Organization. WHO surgical safety checklist; 2020. Available from: https://www.who.int/patientsafety/safesurgery/ checklist/en/ [Accessed 11 May 2020].

6. SURPASS. SURPASS checklist; 2011. Available from: https://www. surpass-checklist.nl/nl/checklijst.html [Accessed 11 May 2020].

7. De Vries EN, Hollmann MW, Smorenburg SM, Gouma DJ, Boermeester MA. Development and validation of the surgical patient safety system (SURPASS) checklist. Qual Saf Health Care 2009;18:121-6.

8. Manataki A, Fleuriot J, Papapanagiotou P. A workflow-driven formal methods approach to the generation of structured checklists for intrahospital patient transfers. IEEE J Biomed Health Inform 2017;21:1156-62.

9. Yi F, Jiang T. Hard frame detection and online mapping for surgical phase recognition. In: Shen D, et al., editors. Medical Image Computing and Computer Assisted Intervention-MICCAI 2019. MICCAI 2019. Lecture notes in computer science. Cham: Springer; 2019, vol 11768. https://doi.org/10.1007/978-3-03032254-0_50.

10. Zia A, Guo L, Zhou L, Essa I, Jarc AM. Novel evaluation of surgical activity recognition models using task-based efficiency metrics. Int J Comput Assist Radiol Surg 2019;14:2155-63.

11. Twinanda AP, Alkan EO, Gangi A, de Mathelin M, Padoy N. Datadriven spatio-temporal RGBD feature encoding for action recognition in operating rooms. Int J Comput Assist Radiol Surg 2015;10:737-47.

12. Feige K, Gollnick I, Schmitz P, Strauss G. The application of surgical procedure manager (SPM): first experience with FESS. Eur Arch Otorhinolaryngol 2017;274:3407-16. 
13. Neumann J, Rockstroh M, Vinz S, Neumuth T. Technical report: surgical workflow and process modeling. Leipzig: Innovation Center Computer Assisted Surgery (ICCAS); 2015. https://doi. org/10.13140/RG.2.1.3280.0725.

14. Freund J, Rücker B. Praxishandbuch BPMN. München: Carl Hanser Verlag GmbH \& Co. KG; 2016. 978-3-446-45054-7.
15. Wiemuth M, Junger D, Leitritz MA, Neumann J, Neumuth T, Burgert 0 . Application fields for the new object management group (OMG) standards case management model and notation $(C M M N)$ and decision management notation (DMN) in the perioperative field. Int J Comput Assist Radiol Surg 2017;12: 1439-49. 